\title{
COMPARISON AND ANALYSIS OF GREEDY FORWARDING USING BNGF METHOD IN DIFFERENT VEHICULAR TRAFFIC SCENARIOS
}

\author{
Ram Shringar Raw \\ SC\&SS, Jawaharlal Nehru University, New Delhi, India \\ rsrao08@yahoo. in
}

\begin{abstract}
A Vehicular Ad hoc Network (VANET) is a most popular application of Mobile Ad hoc Networks (MANETS). A lot of research work around the world is being conducted to design an efficient routing protocol for VANETs. In this paper, we examine the significance of Greedy Forwarding with Border Node based approach for VANETs. We propose a position based routing protocol to forward packet to the border nodes of the forwarding nodeas the next-hop node. This protocol is called Border Node based Greedy Forwarding (BNGF) since it uses border nodes with greedy forwarding. We categorize BNGF as $B N G F-H$ for highway and BNGF-C for city traffic scenario. We have simulated this protocol using NS-2 simulator and evaluated the performance in terms of end-to-end delay and packet delivery ratio. We have compared both the methods for highway and city traffic scenarios. The results clearly show that the end-toend delay for BNGF-C is significantly lower and packet delivery ratio is higher than BNGF-H.
\end{abstract}

\section{KEYWORDS}

MANET, VANET, Routing Protocols, Position Based Routing, Greedy Forwarding, BNGF.

\section{INTRODUCTION}

According to World Health Organization (WHO), millions of people around the world die every year because of vehicular traffic accidents and one fourths of all deaths caused by injury. Also about 50 millions of people are injured in vehicular traffic accidents. Take the metropolitan city Delhi in India for example, where plenty of vehicles like car, truck, buses, motorcycles etc. run on the road at any given time. Delhi has huge population and with respect to population, vehicles population in Delhi is large among all metropolitan cities in India. On an average about 600 new vehicles are added in Delhi every day [1]. Department of transportation annual reports says, thousands of people around the Delhi city die every year because of the vehicular city accidents and many more are injured.

Road safety is a major factor of vehicular traffic management. The exceptional growth in the number of vehicles in the city with limited road space, careless driving and violation of traffic rules caused large number of traffic accidents. Increasing parking demand with limited parking space and unfamiliar with travel related information is an obstruction to the smooth flow of vehicular traffic, especially in crowded and major commercial areas.

Therefore, to reduce large number of vehicular traffic accidents, improve safety, manage traffic control system, and provide important facilities to drivers and passengers with high and reliable 
efficiency, computer networking researchers proposed a new wireless networking concept called Vehicular Ad hoc Network (VANET).

MANETs [2] refer to self-organizing wireless networks consisting of mobile nodes capable to establish communication among them without any fixed infrastructure. Every node in this network acts as router and forwards the message hop by hop. Due to its nature i.e. infrastructurefree environments MANETs can be deployed in emergency rescue, military, airports, sports stadiums, campus, and disaster management. Due to broad applications area of MANETs, researchers paying more attentions in the development of such networks.

VANETs [3] are a special class of Mobile Ad hoc wireless Networks (MANETs). It is currently attracting the extensive attention of research in the field of wireless networking as well as automotive industries. VANETs [4] will provide safer and well-organized road in future by communicating information in timely manner to drivers and concerned authorities. VANETs use short range wireless communication. IEEE 802.11p [5] [6] (modified version of IEEE 802.11a standard protocol) is wireless communication protocol specially designed for VANETs to support safety and non-safety applications. The mobility of nodes in VANETs is very high and it exhibits stronger challenges to researchers. The topology of these networks dynamically changes over time. It causes frequent network partition and makes communication more difficult.

In 1999, the Federal Communication Commission (FCC) allocated a frequency spectrum for vehicle to vehicle and vehicle to roadside wireless communication. The Commission then established the Dedicated Short Range Communications (DSRC) service in 2003. DSRC is a communication service that uses the $5.850-5.925 \mathrm{GHz}$ frequency band $(5.9 \mathrm{GHz}$ band) for the use of public safety and private applications [7]. To facilitate communication within a network, a routing protocol is used to find reliable and efficient routes between nodes so that message delivered between them in timely manner. Routing is responsible for selecting and maintaining routes and forwarding packets along the selected routes. Since the network topology in the VANETs is frequently changing, finding and maintaining routes is very challenging task in VANETs.

In this paper, we propose a novel position-based routing protocol for VANETs called BorderNode based Greedy Forwarding (BNGF) protocol. This routing scheme uses the concepts of border-node of the sender's communication range to maximize packet delivery with minimum delay between source and destination in different vehicular traffic scenarios.

The rest of this paper is organized as follows. Section 2 describes the characteristics of VANET. In section 3, routing protocols for VANET is described. The environment of VANET is described in section 4. The design of proposed routing protocol will be presented in section 5. Section 6 presents the simulation result and performance analysis of the proposed protocol. Finally, we conclude this paper in section 7.

\section{UNIQUE CHARACTERISTICS OF VANET}

VANET have some important characteristics such as nodes forming the networks are vehicles, restricted vehicle movements on the road, highly mobility of vehicles and rapid change in topology, and time-varying vehicle density. We are discussing some unique characteristics [8] that differentiate VANETs form other networks. 


\subsection{Self-Organization}

VANET is self-organizing and adaptive network. Thus a network in VANET may formed or deformed automatically anywhere at any time. The nodes in the network transmit packets with or without the need of a fixed infrastructure.

\subsection{HighlyDynamic Network Topology}

The speed and selection of route defines the dynamic topology of VANET. Roads limit the vehicular network topology to actually one dimension; the road direction.If we assume two vehicles moving away from each other with a speed of $50 \mathrm{~km} / \mathrm{h}(13.88 \mathrm{~m} / \mathrm{s})$ and if the packet transmission range is about $200 \mathrm{~m}$, then the link between these two vehicles will last for only 7.20 seconds $\left(200 \mathrm{~m} / 27.77 \mathrm{~ms}^{-1}\right)$. This defines the VANET has highly dynamic network topology.

\subsection{Unpredictability}

Due to highly node movement and dynamic topology, there is high degree of change in the number and distribution of the nodes in the network at given time instant. Vehicular nodes are usually controlled by pre-built highway, roads and streets. Therefore for the given street map and speed, the future position of the vehicle can be predicted.

\subsection{Infinite Energy Supply}

In VANETs vehicular nodes have plenty of energy and computing power, since nodes are vehicles instead of small handheld devices. Thus vehicular nodes can provide continuous power to their computing and communication devices. As a result, routing protocols do not have to account for methodologies that try to prolog the battery life.

\section{ROUTING PROTOCOLS FOR VANET}

Short duration of communication link between nodes, high vehicles mobility, unpredictable node density, rapid change in topology, and less path redundancy make routing in VANETs relatively challenging. Routing is the important factor for the success of VANET applications since it must efficiently handle rapid network topology changes conditions. Here we discuss the two main category of routing protocols.

\subsection{Ad hoc Routing}

Most of the ad hoc routing protocols such as DSDV (Destination Sequenced Distance Vector) [9], AODV (Ad hoc On demand Distance Vector) [10], and DSR (Distance Source Routing) [11] etc. are designed for general purpose mobile ad hoc networks and they do not maintain routes between nodes unless they are needed. Since the operational principles of VANET and MANET are almost same except the high dynamic topology and frequent networking disconnection due to fast vehicles movement. Therefore some well-known ad hoc routing protocols such as AODV and DSR are partially used in VANETs. In [8], authors evaluated AODV and showed that AODV is unable to quickly find, maintain, and update long routes in VANET. Real world experiment shows packets are extremely lost due to route failure under AODV and it is impossible for a TCP connection to stop its three-way handshake to establish a connection. Thus to meet the VANET challenges, these ad hoc routing protocols need to be modified.

\subsection{Position-based Routing}

Position is one of the most important data for vehicles. In VANET each vehicle wishes to know its own position as well as its other neighbor vehicle's position. A routing protocol using position 
information in known as the position based routing (see Figure 1). Position based routing protocols need the information about the physical location of participating vehicles be available. This position can be obtained by periodically transmitted control messages or beacons to the direct neighbors. A sender can request the position of a receiver by means of a location service.

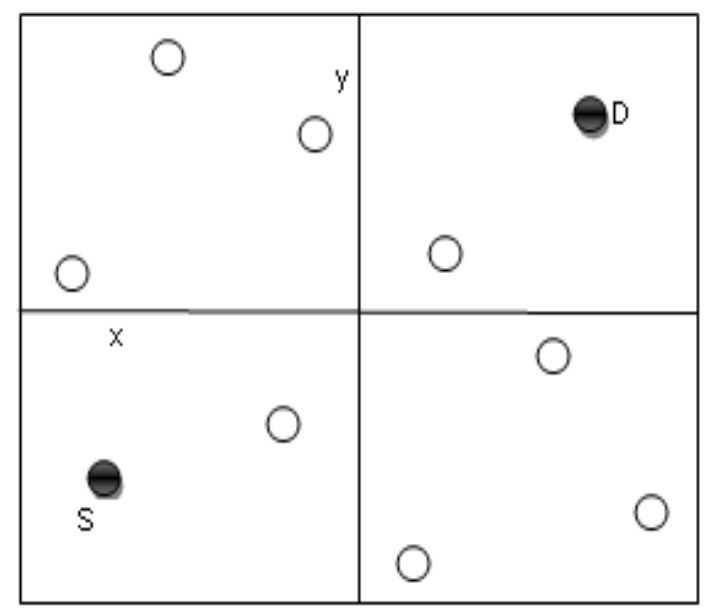

Figure 1. Position-based routing scheme

In VANETs, route is composed of several pair of vehicles (communication links) connected to each other from the source vehicle to the destination vehicle. If we know the current information of vehicles involved in the routes, we can predict their positions in the near future to predict the link between each pair of vehicles in the path.

Various approaches have been recommended to improve the throughput and the efficiency of position based routing protocols [8] [12] [13] in highways or rural areas and city or urban areas for linear and non-linearnetwork topology. Next-hop forwarding method like greedy forwarding scheme for linear network does not support well in highly mobile ad hoc network such as vehicular ad hoc network. Therefore, other position based protocols such as DIR, MFR, GEDIR, B-MFR etc. have been used for VANET to improve its performance for non-linear network in a high vehicular density environment.

A DIrectional Routing (DIR) [14] (referred as the Compass Routing) is based on the greedy forwarding method in which the source node uses the position information of the destination to calculate its direction. Then the message is forwarded to the nearest neighbor having direction closest to the line drawn between source and destination. Thus a message is forwarded to a neighbor such that the angle between the source and the selected node is minimized.

MFR (Most Forward within Radius) [15] [16] is a well-known method for finding a route in a network by utilizing position information of nodes. The neighbor with the greatest progress on the straight line is chosen as next hop for sending packets further. Therefore MFR forwards the packet to the node that is closest to the destination node in an attempt to minimize the number of hops. A GEDIR (GEographic DIstance Routing) [15] is a loop free location based routing algorithm. It is the variant of greedy routing. In GEDIR, a source node forwards packets to its neighbor node that is closest to the destination node. 
DIR, MFR, and GEDIR, protocols can be further improved by utilizing farthest one-hop node in a dense and highly mobile network. In [16], authors proposed a routing protocol that uses BorderNodes with maximum projection. We call this protocol Border-node based Most Forward within Radius (B-MFR). The B-MFR utilizes the border-node to avoid using interior nodes within the transmission range for further transmitting the packet. This method selects the border-node as a next-hop node for forwarding packet from source to destination. In this method, a packet is sent to the border node with the greatest progress as the distance between source and destination projected onto the line drawn from source to destination.

\section{ENVIRONMENTS OF VANET}

In this section the VANET environments characteristics are described (see Figure 2). In rural areas node density is very low, terrain effects may be important, inter-vehicle spacing can be large and there is very less or no availability of central administration or fixed infrastructure. Thus, in rural areas nodes of the vehicular ad hoc network are often disconnected and this situation may cause a loss of messages. On highways the traffic pattern is totally different, where vehicles are driven at high speeds following a road without crossovers or traffic lights. Traffic density and infrastructure deployment are highly highway-dependent, even though it is probable that road side units (RSUs) will be encountered with which the vehicles can communicate.

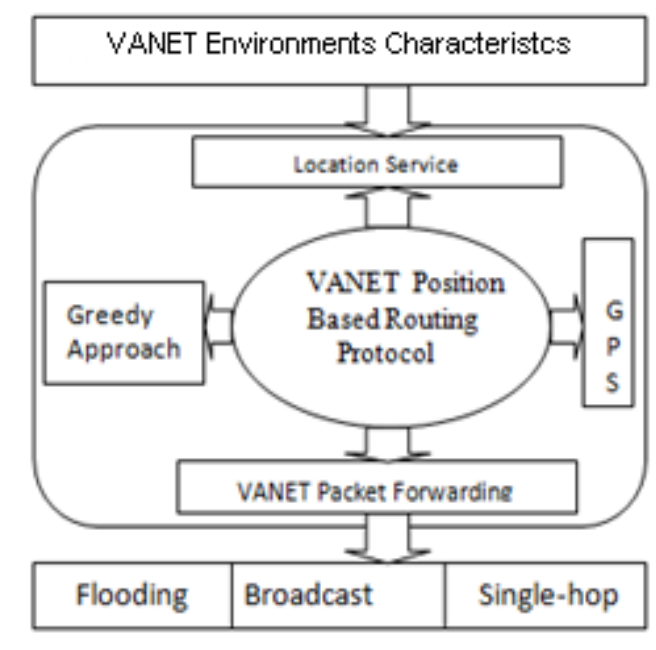

Figure 2. Major components of VANET environments

In VANETs [17] most of the research work has focused on urban roadway conditions, where the number of vehicles are large (high density of vehicles) which makes it is easier to find a path between source to destination and may cause contention and collisions. In urban areas, the spacing between vehicles is small and fixed communication infrastructure is available that increases the communication capability of the smart equipped vehicles. In urban areas, roads are located to each other, there exist obstacles, such as buildings and advertisement walls, which stop wireless signals from travelling between roads. A road can be considered as a straight line or a small-angled curve and vehicles on the road can be considered as points on the same line. 


\section{PROPOSED WORK}

\subsection{Assumptions}

The BNGF protocol design is based on the following assumptions [18].

- $\quad$ Border nodes for forwarding packets

- $\quad$ Hello (beacon) control message for next-hop neighbors

- $\quad$ Vehicles are equipped with GPS receiver, electronic digital maps and sensors

- $\quad$ Communication between vehicles using wireless ad hoc network

- $\quad$ No other communication infrastructure

- $\quad$ Maximum forwarding distance mar vary

- $\quad$ Forwarding direction towards destination

- $\quad$ Message based communication

\subsection{Procedure of Border Node Selection}

A node in the network has a set of one-hop nodes within its transmission range. These one-hop nodes are called neighbor nodes. The one-hop neighboring nodes are divided into two groups interior nodes and border nodes. A border node [18] [19] [20] is defined as a peripheral node, whose distance from the central node is exactly $R_{0}$, which is equal to the maximum transmission range $\mathrm{R}$ of the central node. Therefore, the border node lies furthest away from the central node within its transmission range (see Figure 3).

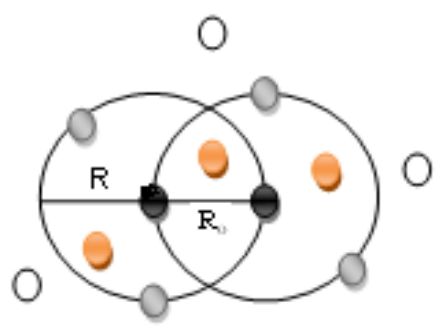

(a)

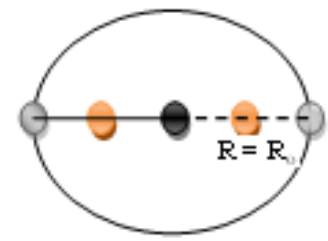

(b)

$$
\begin{gathered}
\text { = Source Hode } \quad Q=\text { Border Hode } \\
=\text { Interior Hode } \quad Q=\text { Onter Hode } \\
R=\text { Radius of Circle }, R o=\text { Max Transmiss ion Ramg }
\end{gathered}
$$

Figure 3. Border node architecture

\subsection{Border Node based Greedy Forwarding Method (BNGF)}

Procedure of the proposed selection method is described in this section. Next-hop forwarding method like greedy forwarding scheme (see Figure 4) for linear network does not support well in highly mobile ad hoc network such as vehicular ad hoc network. Therefore, other position based protocols such as MFR, GEDIR, Compass routing, etc. have been used for VANET to improve its performance for non-linear network in a high vehicular density environment. 


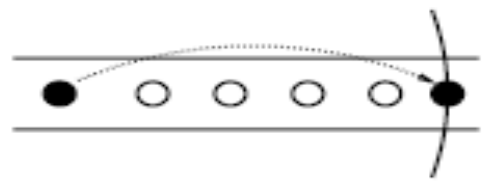

Figure 4. Greedy forwarding

These protocols can be further improved by utilizing farthest one-hop node in a dense and highly mobile network. In this paper, wehave proposed a routing protocol that uses Border-Nodes that is closest to destination. The BNGF utilizes the border-node to avoid using interior nodes within the transmission range for further transmitting the packet. This method selects the border-node as a next-hop node for forwarding packet from source to destination. In this method, a packet is sent to the border-node that is closer to destination in both linear (highway) and non-linear (city) highly dynamic network.

\subsubsection{BNGF in Highway (BNGF-H)}

The BNGF-H protocol is designed for sending messages from one node to other node by using unicast forwarding. This is likely to be an appropriate method for routing in highway VANETs by considering routes over vehicles driving on the straight line in the same direction. VANET in highway can be characterized as partially connected network with low node density and high node mobility. Therefore designing of routing protocols for highway VANET scenarios is challenging. This method is well suited for vehicle to vehicle communications in rural and remote areas highways.In BNGF-H an ideal node to forward a packet would be the node located at the border of the communication range of the source node. The border node is selected based on direct one-hop neighbor information using unicast forwarding.

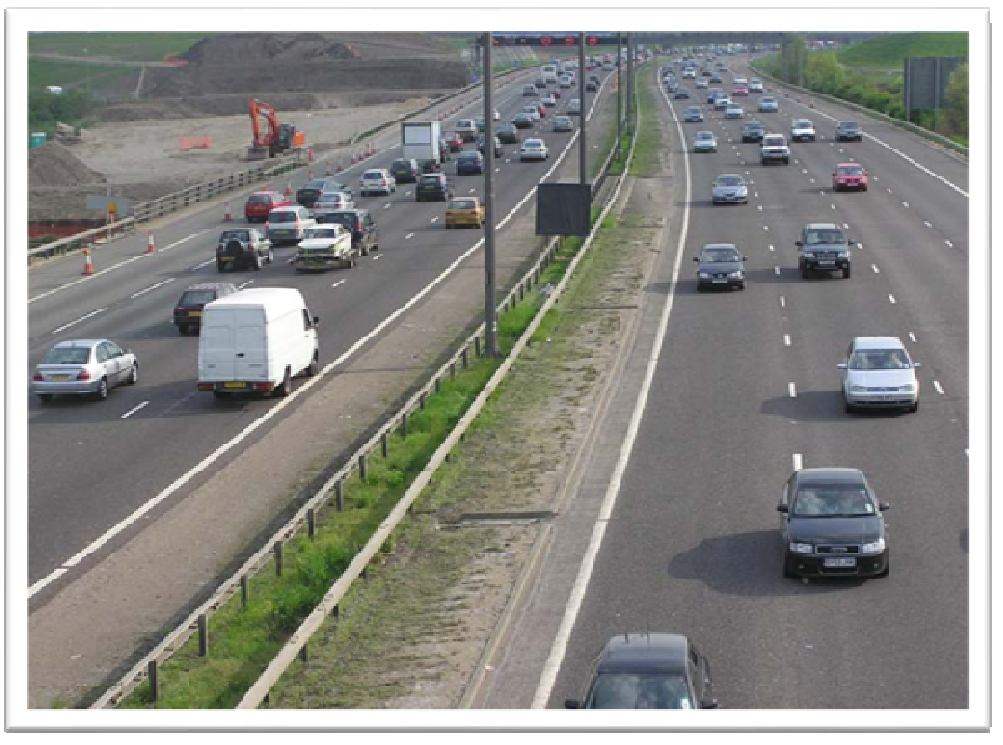

Figure5. Highway traffic scenario 
Highway scenario (see Figure 5) may have single or multiple lane, uni or bi-directional way. Destination is a moving vehicle, located ahead or behind of the source vehicle in the same lane . This method is more suitable for highway or rural traffic environment where nodes are evenly distributed on the straight line within the transmission range in the networks. In Figure 6, node $A$ is the border node of the communication range of source node $S$. Source node $S$ selects node $A$ as the next-hop node for forwarding packet further because node $A$ is the farthest node (border node) within its transmission range. When node $A$ receives the message, it uses the same method to selects the next-hop border node and forward the packet further. In this way, finally node $B$ is selected as a next-hop border node for forwarding packets to destination.

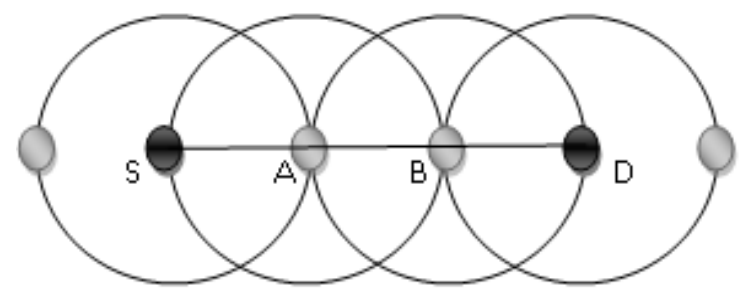

Figure 6. BNGF-H forwarding method

Although it is not necessary (less probability) that nodes on the border of the transmission range of each forwarding node will be available all the time during packet transmission from source to destination. If such situation comes, this BNGF-H method will give better performance than other greedy forwarding method as the number of hops from the source to destination will be minimized.

\subsubsection{BNGF in City Scenario (BNGH-C)}

Generally city scenario consists of several vertical and horizontal streets as well as many crossings and junctions. The traffic density is very high in city scenario for VANET due to a potentially large-scale network. VANET in city scenario has several characteristics such as signal reception is more difficult due to radio obstacles, scalability is very high due to open network environment, neighboring network density always changes depending upon the time and area. Due to these distinctive characteristics the design of routing is very challenging in city vehicular scenario. Despite the challenges above, city VANET has many advantages like vehicles utilizes the full use of Global Positioning System (GPS) receiver and city digital maps without having resource limitations which can be used to make better routing decisions. The goal of routing protocol in city scenario is to avoid collisions caused by hidden vehicles and sending message in all directions. The packet should be delivered with high successful rate. 


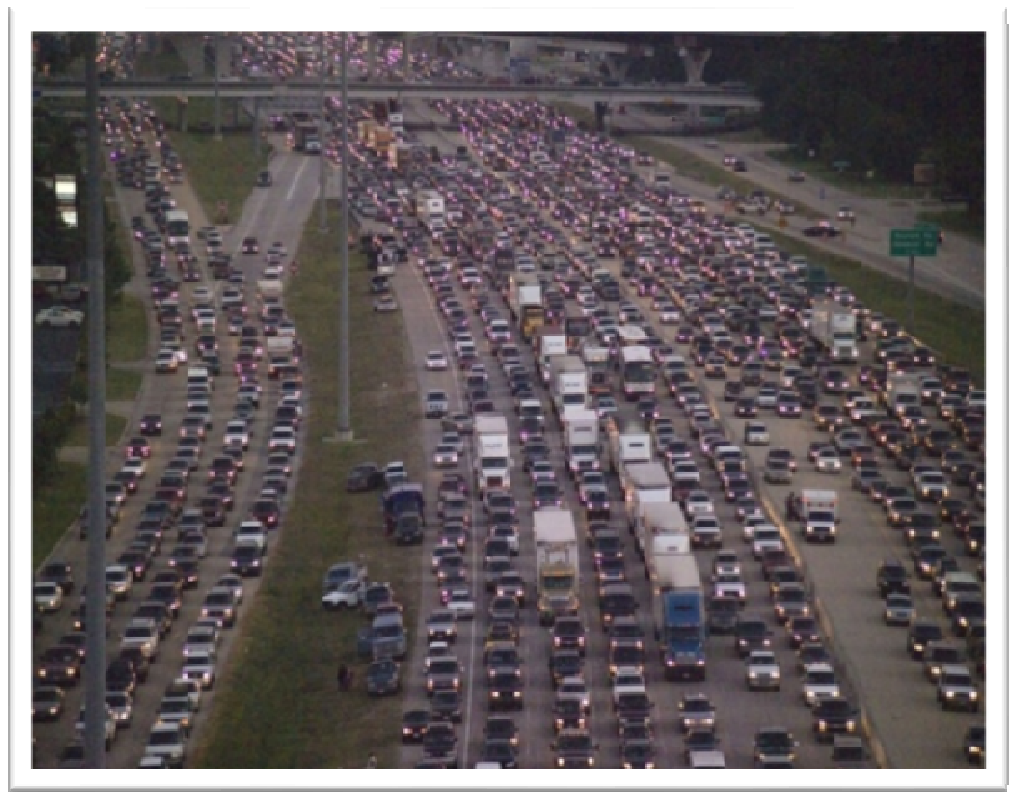

Figure 7. City traffic scenario

City traffic scenario (see Figure 7 ) consists plenty of vehicles, lot of vertical and horizontal roads, and omni-directional way. Destination is a moving vehicle, located in any directions at any point of the source vehicle. Road Side Unit (RSU) is available along the roadway to relay the message in all directions. In BNGF-C, the source node tries to select the furthest node (border node) in the direction of the destination for forwarding packet from source to destination. This method is more suitable for city or urban traffic environment where nodes are distributed unevenly within the transmission range in the network.

In Figure 8, node $S$ and $D$ are source and destination nodes. Source node $S$ has three border nodes. Among these border nodes, source node $S$ selects the border node $A$ as the next-hop node that is closest to the destination node $D$ for forwarding packet further. When node $A$ receives the packet, it uses the same procedure to select next-hop border node. In this way, finally node $B$ is selected as a next border node for forwarding packets to destination in the network.

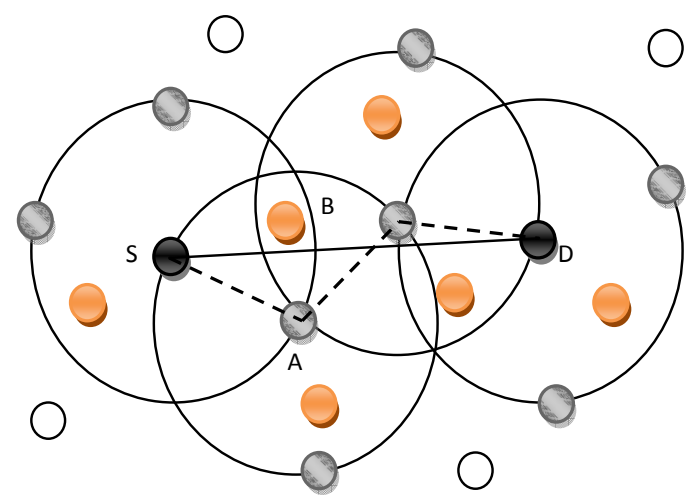

Figure 8. BNGF-C forwarding method 


\section{SIMULATION RESULTS AND PERFORMANCE ANALYSIS}

To evaluate the performance of BNGF protocol as BNGF-H and BNGF-C, it is implemented using NS-2 simulator and simulations are conducted. BNGF-H compared with BNGF-C position based routing protocol in vehicular environment. Based on the simulation parameters given below, the protocol has been simulated with a variable transmission range from $200 \mathrm{~m}$ to $1000 \mathrm{~m}$. We consider highway traffic scenario where vehicles are moving on the straight line and a city traffic scenario where vehicles are moving in every direction.

Table 1. Simulationsetup

\begin{tabular}{|l|l|}
\hline Parameter & Values \\
\hline Simulation time & $200 \mathrm{~s}$ \\
\hline Simulation area & $2000 \mathrm{~m} * 2000 \mathrm{~m}$ \\
\hline No. of Vehicles & $30,50,70,90,110$ \\
\hline Vehicle's Speed & $30-50 \mathrm{Km} / \mathrm{h}$ \\
\hline Transmission Range & $200 \mathrm{~m}-1000 \mathrm{~m}$ \\
\hline No. of Packet Senders & 30 \\
\hline Packet Size & $512 \mathrm{bytes}$ \\
\hline CBR (packets/sec) & $0.1,0.2,0.4,0.6,0.9,1$ \\
\hline Vehicle Hello Interval & $0.20,0.40,0.80$ \\
\hline MAC Protocol & IEEE 802.11, DCF \\
\hline
\end{tabular}

The IEEE 802.11 DCF (Distributed Coordinated Function) is used as the MAC protocol. We use a $2000 \mathrm{~m} \times 2000 \mathrm{~m}$ square area for simulation. Network size is represented by the number of vehicles. The speed of vehicles varies from $30-50 \mathrm{~km} / \mathrm{h}$. The traffic density is not uniform and it depends on the number of vehicles chosen in the given area. Among all the vehicles, 30 pairs of source-destination are chosen randomly to send packets. The packet transmission density can be adjusted by setting different CBR rates with a packet size of 512 bytes. A simulation runs for 200 seconds and we have taken average of 10 simulation runs. Hereafter the terms node and vehicle are used interchangeably.

\subsection{End-to-End Delay (EED)}

This is the average delay between source and destination node for all successfully delivered data packets. In Figure 9, the end-to-end delay for BNGF-C is significantly lowers than BNGF-H. Further, BNGF-C has comparatively small end-to-end delay when number of vehicles becomes more. Therefore from this figure, we can observe that BNGF-C outperform BNGF-H in terms of end-to-end delay. 


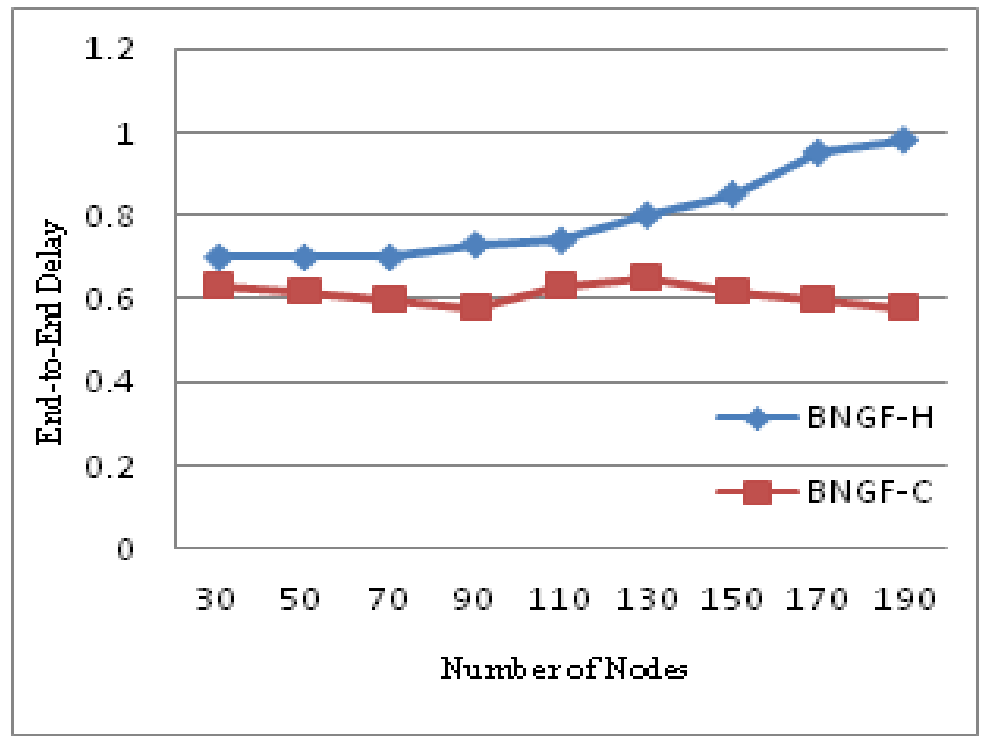

Figure 9. End-to-end delay

This improved performance of BNGF as BNGF-H and BNGF-C in different traffic scenarios can easily be explained by understanding the significance of using border nodes in our protocol as next hop forwarding node. Therefore, in BNGF-C, the time taken to deliver the packet from source to destination (end-to-end delay) is reduced. Further, in BNGF-C as the node density increases, the probability of presence of border node increases as compared to BNGF-H. This gives higher rate of successful deliveries and reduction in number of retransmission. This improves the end-to-end delay that is evident from the figure as the end-to-end delay for BNGF-C grows slowly as the number of nodes increases.

\subsection{Packet Delivery Ratio (PDR)}

Packet delivery ratio is the ratio of the packets that successfully reach the destination.

$P D R=\frac{\text { Total number of packets delivered }}{\text { Total number of packets transfered }} \times 100$

Here we compare the performance of BNGF-H and BNGF-C in terms of packet delivery ratio. From the Figure 10, we can see how packet delivery is affected by the packet transmission density and vehicular traffic density. In case of low vehicle density, very few vehicles will be available within the transmission range for next-hop selection along a particular path. When the vehicle density is more, the connectivity is much better. In this case all routing methods achieves better delivery ratio, since more vehicles can be met to forwards packets. In BNGF, a node will forward packet to the next-hop border node of its transmission range which is moving towards the destination. The packet delivery ratio is directly proportional to the vehicle density. As shown in Figure 10, BNGF-C outperforms BNGF-H in terms of packet delivery ratio when the vehicle density is high. 


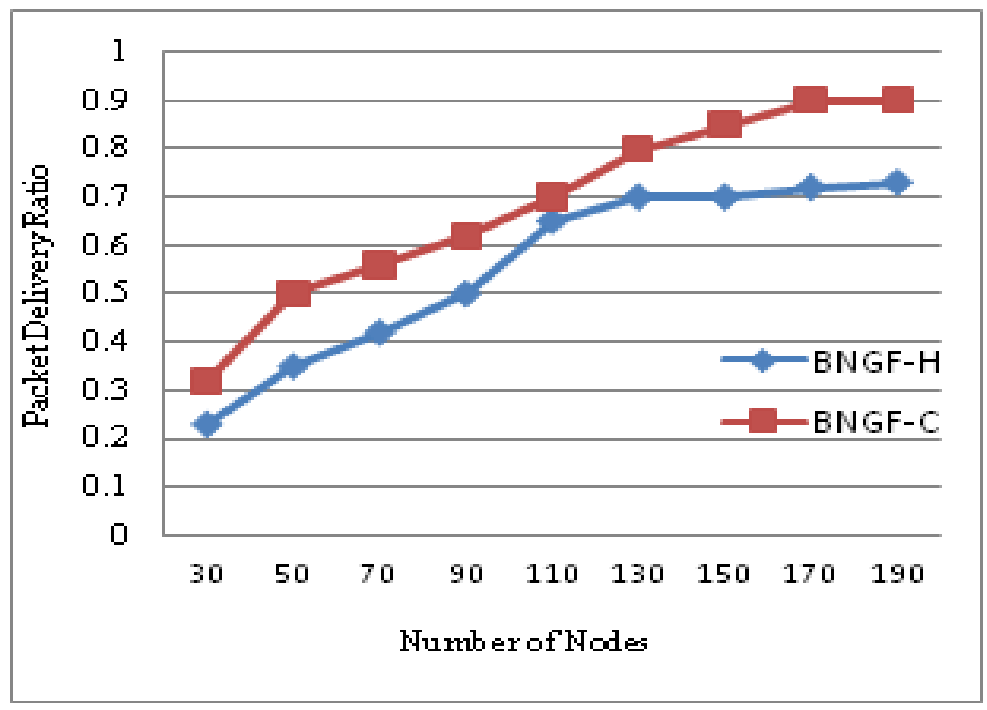

Figure 10. Packet delivery ratio

\subsection{Packet Delivery Ratio Vs. Radio Transmission Range}

Figure 11 shows that when the transmission range increases, the packet delivery ratio initially increases rapidly in both the cases, BNGF-H and BNGF-C. After the transmission range reaches about 400 meter, the packet delivery ratio remains constant at about $90 \%$ in BNGF-C and at about $68 \%$ in BNGF-H and then gradually increases $95 \%$ and $73 \%$. When we compare BNGF-H and BNGF-C, BNGF-C outperforms BNGF-H as the transmission range increases the packet delivery ratio is also increases proportionally. Thus BNGF-C gives better performance than BNGF-H in terms of packet delivery ratio when the radio transmission range increases.

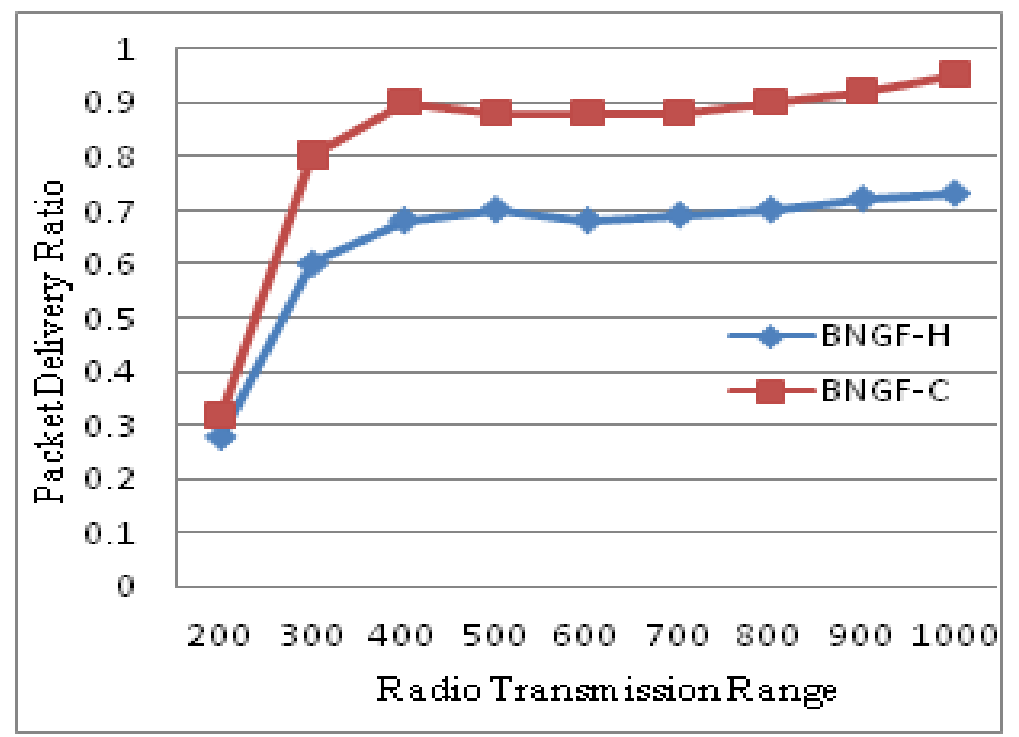

Figure 11. Packet delivery ratio Vs. radio transmission range 


\section{CONCLUSION AND FUTURE WORKS}

In this work, we have characterized the VANET. By using the unique characteristics of VANET, we have proposed a new Border Node based Greedy Forwarding method (BNGF). BNGF protocol is designed to find robust paths to forward packet with minimum delay. Furthermore, we have categorized the BNGF as BNGF-H and BNGF-C for two different traffic scenarios, highway and city for VANETs. The simulation result shows that BNGF-C outperforms BNGF-H in terms of end-to-end delay and packet delivery ratio. Therefore, in city scenario BNGF-C gives higher rate of successful deliveries and reduction in number of retransmission. This improves the end-toend delay as the number of nodes increases.

As for future works, VANETs are expected to be very attractive in near future, so VANETs needs more research which could lead to further improvements in vehicular ad hoc routing. Our aim is to have an efficient position based routing protocol, which works well in every traffic scenario.

\section{REFERENCES}

[1] Rites LTD, (1994) “Urban Environmental Engineering”, Household Surveys, Delhi.

[2] G. Jayakumar, and G. Gopinath, (2007) "Ad Hoc Mobile Wireless Networks Routing Protocols A Review”, Journal of Computer Science 3 (8): 574-582.

[3] H. Moustafa and Z. Yan, (2009) "Vehicular networks: Techniques, Standards, and Applications", CRC Press.

[4] S. S. Manviand M. S. Kakkasageri, (2008) "Issue in Mobile Ad hoc Networks for Vehicular Communication”, IETE Technical Review, Vol. 25, No. 2.

[5] Task Group p "IEEE 802.11p Wireless Access for Vehicular Environment”, Draft Standard, http://grouper.ieee.org/groups/802/11/.

[6] Q. Chen, D. Jiang, V. Taliwal, and L. Delgrossi, (2006) "IEEE 802.11 based Vehicular Communication Simulation Design for NS-2”, VANET' 06, Los Angeles, California, USA.

[7] D. Jiang, V. Taliwal, A. Meier, W. Holfelder, and R. Herrtwich (2007) "Design of 5.9GHz DSRCBased Vehicular Safety Communication".

[8] F. Li and Y. Wang, (2007) "Routing in Vehicular Ad Hoc Networks: A Survey", IEEE Vehicular Magazine.

[9] C. E. Perkins and P. Bhagwat, (1994) "Highly dynamic destination sequenced distance-vector routing (DSDV) for mobile computers", Computer Communication Review, pp. 234-244.

[10] C. E. Perkins and E. M. Royer, (1998) “Ad-hoc On-Demand Distance Vector Routing”.

[11] D. Johnson and D. Maltz, (1996) "Dynamic Source Routing in Ad-hoc Wireless Networks", Computer Communications Review - Proceedings of SIGCOMM' 96.

[12] A. Takano, H. Okada, and K. Mase, (2007) "Performance Comparison of a Position-Based Routing Protocol for VANET", IEEE.

[13] I. Stojmenovic, (2002) "Position-Based Routing in Ad Hoc Networks", IEEE Communications Magazine. 
[14] E. Kranakis, H. Singh, and J. Urrutia, (2000) “Compass Routing on Geometric Networks”.

[15] S. Giordano, I. Stojmenovic, and L. Blazevic, (2003) "POSITION BASED ROUTING ALGORITHMS FOR AD HOC NETWORKS: A TAXONOMY”.

[16] R. S. Raw and D. K. Lobiyal, (2010) "B-MFR Routing Protocol for Vehicular Ad hoc Networks", IEEE, ICNIT- 2010, Manila, Philippines.

[17] Z. Guoqing, M. Dejun, X. Zhong, Y. Weili, and C. Xiaoyan, (2008) "A Survey on the Routing Schemes of Urban Vehicular Ad Hoc Networks", Proceedings of the 27th Chinese Control Conference, China.

[18] M. Zhang and R. S. Wolff, (2009) "Routing Protocols for Vehicular Ad Hoc Networks in Rural Areas".

[19] M. Zhangand R. S. Wolff, (2010) "A Border Node Based Routing Protocol for Partially Connected Vehicular Ad Hoc Networks”, Journal of Communications, VOL. 5, NO. 2.

[20] R. S. Raw, S. Kumar, and S. Das, (2010) “An Efficient Position-Based Routing Protocol for Vehicular Ad hoc Network in City Traffic Scenarios”, RISTEP-2010, NIT Srinagar, India.

\section{Author}

Ram Shringar Raw received his B. E. (Computer Science and Engineering) from G. B. Pant Engineering College, Pauri-Garhwal, UK, India and M. Tech (Information Technology) from Sam Higginbottom Institute of Agriculture, Technology and Sciences, Allahabad (UP), India in 2000 and 2005, respectively. He is pursuing Ph.D (Computer Science) from School of Computer and Systems Sciences, Jawaharlal Nehru University, New Delhi, India. He is currently working as Assistant Professor at Computer Science and Engineering Department, G. B. Pant Engineering College, Uttarakhand Technical University, since 2001. His current research interest includes

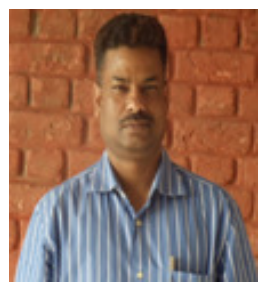
Mobile Ad hoc Networks and Vehicular Ad hoc Networks. Mr. Raw has published papers in International Journals and Conferences including IEEE, Springer, Inder Science and AIRCC. 